\title{
Maturity Model for Improving Virtual Engineering in Small and Medium-Sized Enterprises
}

\author{
Tobias Bensiek and Arno Kuehn \\ Heinz Nixdorf Institute, University of Paderborn, Germany \\ \{tobias.bensiek, arno.kuehn\}@hni.upb.de
}

\begin{abstract}
This paper introduces the VPS-Benchmark - a maturity model for performance evaluation and improvement in small and medium-sized enterprises (SMEs) with a special focus on virtual engineering. By giving a brief overview on existing approaches for performance evaluation and improvement we point out the demand for a new maturity approach specialized on the requirements of SMEs. The new maturity model will then be introduced. After describing the basic concept of the model, we focus on the performance improvement strategy resulting from its application. The model offers a step by step performance improvement to SMEs. This improvement is based on a so called ACT-concept that motivates SMEs to deal more intensively with virtual engineering and to exploit its benefits by implementing concrete measures.
\end{abstract}

Keywords: performance evaluation and improvement, process maturity models, product development process, virtual engineering.

\section{Introduction}

Successful companies in mechanical engineering and related industrial sectors are characterized by efficient and effective processes. Particularly, the product development process is of special interest, as it determines the features of the future product and about $80 \%$ of product costs.

Methods and tools of Virtual Prototyping and Simulation (VPS) become more and more accepted in these processes. Virtual prototyping means creating and analyzing computer models of products in development. This reduces the number of physical prototypes, improves communication in development processes and helps to avoid design errors. That way, companies save time and money [1],[2].

In the past, lots of domain-specific methods and tools, for VPS e.g. CAD (computer-aided design) and FEM (finite element method), emerged. The transition from a traditional product development, based on physical prototypes, to an integrated virtual engineering, based on VPS, is expensive and often characterized by costly failures. The integration of these tools into the processes and the PLM-infrastructure (product lifecycle management) of the company is a success factor in the development of complex technical systems [3]. However, especially small and medium sized enterprises (SMEs) often run isolated applications that are insufficiently integrated into the development process and the PLM-infrastructure. This means that only fractions of the benefits of virtual engineering are exploited. 
In order to improve their product development processes, SMEs need support for planning and optimization of their product development processes. Key points of improvement are both, an objective evaluation of current performance as well as a coherent conception for improvement. Maturity models target at these points. [4]

This paper starts with specific requirements arising out of the need for maturity models in SMEs. By giving a brief overview on existing approaches for performance evaluation and improvement we point out the demand for a new approach specialized on the requirements of SMEs. The new approach with its focus on an integrated virtual engineering will then be introduced. After describing the framework of this approach, we focus on the performance improvement strategy resulting from its application.

\section{Requirements for Maturity Models in SMEs}

SMEs not only lack the experience in VPS-technologies but also the knowledge about their potential benefits. Additionally, the introduction and integration of those technologies in terms of an integrated virtual engineering requires extensive planning and thus financial and human resources that are often missing. While large companies maintain own planning departments, SMEs have little time to perform process improvement initiatives in addition to the dominant day-to-day business [5]. Out of these circumstances specific requirements arise for the application of maturity models in SMEs. These requirements were verified in several interviews with SMEs, vendors and consultants.

- Pragmatic Performance Evaluation with Low Effort: Time to investigate possible approaches and the extensive bureaucracy are barriers to use assessment tools for performance improvement [6]. SMEs neither have the time nor the money to get into complex models for performance evaluation and improvement. Thus, an easily learnable and practicable model is necessary. External experts should be at least to some extent unnecessary. Additionally, time for application should be as short as possible and not exceed a few days.

- Consideration of Performance Improvement: Due to the lack of knowledge about potential benefits of VPS, SMEs need concrete recommendations for performance improvement. Thus, the approach has to support the interpretation of the performance evaluation.

- Definition of a Company-Specific Target State: Especially for SMEs, it must not be economical to achieve the highest performance level. Considering a SME with only a few engineers, the cost-benefit-ratio of implementing and maintaining a PDM-system is questionable. Ignoring other possible influences, managing data within well-organized file structures would be adequate. The example demonstrates that recommendations have to aim at an individual target state.

- Based on Best Practices: Other barriers to use of assessment tools in industry are the general high resistance towards change and the missing interest of the senior management [6]. To achieve acceptance among the management and the employees, it is important to base all resulting performance improvement recommendations on proven practices from industry and science.

The model presented below has been created considering the guidance for developing maturity models of de Bruin [7], Christiansen [8], Kohlegger [9] and Maier [10]. 


\section{Performance Evaluation and Improvement Using Maturity Models}

According to current estimates, more than 200 different maturity models are available [11]. Therefore, structuring the state of the art of performance evaluation and improvement is necessary.

Selecting an optimal model depends on two main factors. First, the model has to fit the area of interest. Existing models cover a wide spectrum of different applications. CMMI-DEV (Capability Maturity Model Integration for Development) e.g. focuses on product development, ITIL (IT Infrastructure Library) on IT management and SPICE (Software Process Improvement and Capability Evaluation) on software development [11]. Also for virtual engineering and PLM there are already existing models, such as "ENGINEERING produktiv!"1 or Batenburg [12].

Second, the methodology of the maturity model must match the user's requirements. The methodology has an impact on features such as the consideration of performance improvement, the assessment system and the effort for training [8].

Comparing CMMI-DEV and PEMM (Process and Enterprise Maturity Model) demonstrates that these features differ heavily between the available models. Introducing CMMI-DEV in a company requires intensive training of staff and involvement of different divisions [13], [14]. A complete implementation of CMMI-DEV often requires months (depending on company size). In contrast, PEMM is a very pragmatic approach that can be applied with minimal effort and without trained staff. In a selfassessment the maturity levels can be determined within days [15].

Due to the variety of these models, selecting an appropriate maturity model is difficult. Therefore, the Heinz Nixdorf Institute developed a classification of models for performance evaluation and improvement [8]. Mettler [16] and Van Looy et al. [17] describe alternative classification approaches. One type of maturity models are selfassessments. Self-assessment tools allow to get a quick (a few days) impression about the current performance of a specific area of the company. They are interesting due to the low effort resulting from its pragmatic application. This tools best fit the requirements described in section 2 .

The disadvantage of these models is the missing consideration of performance improvement. Röglinger et al. [18], [19] describe the missing of a defined mechanism to select improvement measures and derive an improvement strategy. Most of these models only offer a visualization of results in diagrams, showing the current performance. Hence, deriving concrete measures to improve performance requires expert knowledge. As the SMEs often lack this knowledge, they are reliant on specialists supporting the development of a performance improvement strategy. Therefore, an approach is needed that on the one hand is based on a pragmatic self-assessment and on the other hand gives concrete measures for performance improvement. As already mentioned in section 2, the highest level of performance must not be the most economical one for a SME. Thus, the approach also has to support the definition of a company-specific target state.

\footnotetext{
${ }^{1}$ Internet portal of the initiative ENGINEERING produktiv!: http://www.engineering- produktiv.de
} 


\section{A Maturity Model for Process Improvement in SMEs}

In the following, we introduce the VPS-Benchmark - a maturity model for performance evaluation and improvement focusing on virtual engineering. The model allows a quick and easy evaluation and identification of potential process improvements. Additionally, the strategic planning of VPS-usage is supported. The target group are SMEs in mechanical engineering and related industrial sectors. In section 4.1 , we explain the basic concept of the maturity model. In section 4.2, we detail the application of the model and the performance improvement.

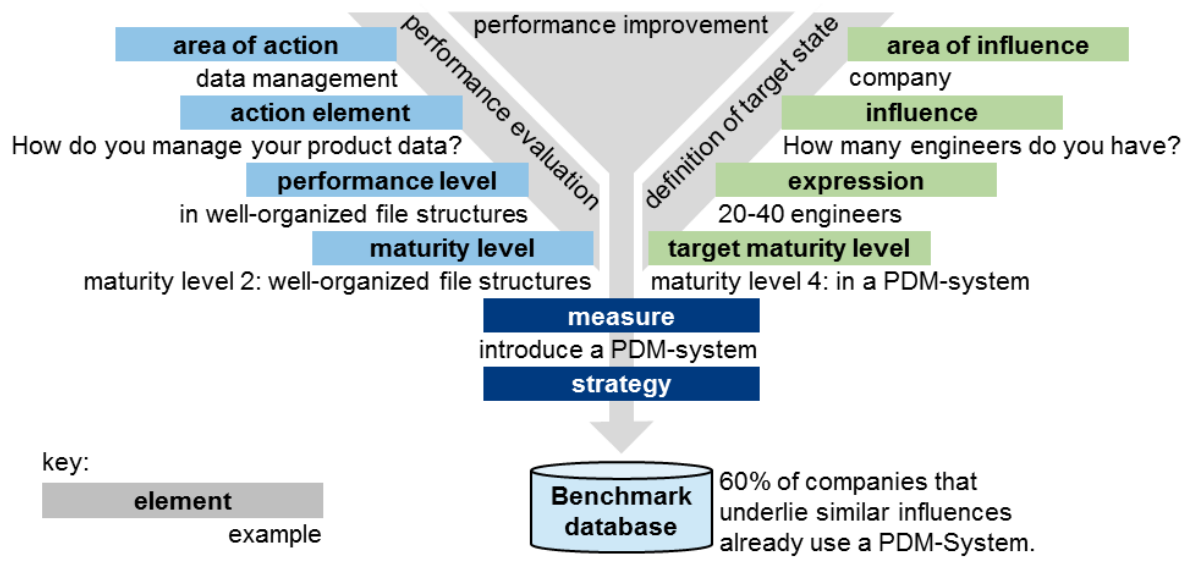

Fig. 1. Framework of the maturity model

\subsection{Basic Concept of the Maturity Model}

The procedure of the VPS-Benchmark is structured in 3 parts (figure 1): performance evaluation, definition of target state and performance improvement. In the following, we describe the framework of the maturity model. Therefore, we use an intuitive example, the introduction of a PDM-system.

The left wing, the performance evaluation, consists of 4 elements. These elements are closely related to the basic structure of typical maturity models as described in [8].

- Areas of action categorize the area of interest according to superior criteria, such as organization and technology. They ensure that all relevant facets are considered without having a one-sided perspective. In the context of VPS we consider 5 areas of action. An example is data management.

- Action elements are performance indicators for the particular area of action. For each area of action there are 20 to 50 action elements. An example is the application of a PDM-system. Action elements are formulated as questions, such as: „How do you manage your product data?“” 
- Performance levels are the predetermined answers to the questions of the action elements. They indicate in which stage of development the action element is established. A low performance level would be well-organized file structures.

- All performance levels are associated with maturity levels. Maturity levels express the performance of an organization in an objective and measureable manner. Consequently, a high maturity level means a high developed action element and thus a good performance in the particular area of action.

Beyond the current maturity level, the definition of the target state is required for deriving a strategy for performance improvement. A company-specific target state is defined in the right wing of the framework.

- Areas of influence are the equivalent to the areas of action in performance evaluation. They ensure that all relevant aspects are considered, which have an impact on the definition of the target state. Areas of influence in the context of VPS are the company, its products and its business environment.

- Influences and its expressions indicate in which stage of development the action element should be established. Similar to the action elements and performance levels they are formulated as questions with predetermined answers. In the example, we win the information that the company has 20-40 engineers.

- Out of the information given by the influences and its expressions, we can derive a recommendation for the target state expressed as target maturity level. In the example, the use of a PDM-system would be recommended to optimize the data management. The target maturity level is the equivalent to the maturity level in performance evaluation.

The bigger the difference between target maturity level and initial maturity level, the higher the need for action. The question arising is how to achieve the desired target state. This question is answered by the third part, the performance improvement.

- The comparison between target maturity level and initial maturity level provides recommendations for improvement in form of concrete measures. In case of the given example the measure would be introduce a PDM-system.

- As there can be a lot of resulting measures, a strategy for their implementation is needed. This performance improvement strategy must include a prioritization of measures in terms of a cost-benefit-evaluation.

All results are collected in a so called benchmark-database. This database allows a benchmark between companies that underlie similar influences. Having enough data sets, the conclusion could be: $60 \%$ of the companies that underlie similar influences already use a PDM-system.

The basic concept of the model is described in figure 2. We differentiate between the development and the application of the maturity model. Both are interconnected by an interactive questionnaire, which will be available on an internet portal ${ }^{2}$.

\footnotetext{
${ }^{2}$ The internet portal www . viprosim. de promotes the usage of VPS especially in SMEs.
} 
experts

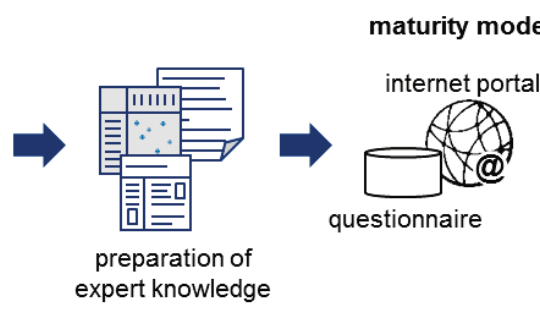

expert knowledge

development of the maturity model

SMEs

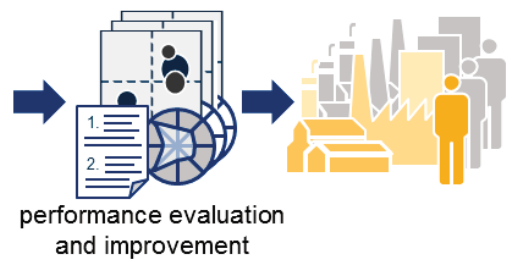

application of the maturity model

Fig. 2. Basic concept of the maturity model

The development of the maturity model is based on expert knowledge that is prepared and converted for the interactive questionnaire. The elements of the framework and their interrelations represent the expert knowledge. The knowledge is gathered in expert-workshops. We differentiate between three types of experts:

- Vendors are specialists for dedicated VPS-tools, such as CAD-, PDM- or CAEsystems. They know best about the functional range of their tool and its potential benefits.

- Consultants can compare various product development processes and the advantageous application of VPS. They are specialists for optimal process integration.

- Commendable SMEs provide best practices. They know best about their particular requirements.

The expert knowledge is prepared via matrices, profiles and lists. A detailed description of the preparation and analysis is given in [19].

\subsection{Application of the Maturity Model}

The SME applies the maturity model by answering the questionnaire. To cover all relevant facets of virtual engineering, the questionnaire should be completed in a team including representatives from engineering, sales, marketing, production and documentation. In a first step, the SME answers questions to various topics of VPS. Herewith, the current state expressed as maturity level is evaluated. In a second step, questions about the company, its products and its business environment are asked. This information determines a company-class and a company-specific target state. A comparison between current and target state delivers the need for action. To close this gap the SME has to ACT (accept - comprehend - tap). In figure 3 the path from identifying the need for action to improvement is described as the ACT-concept. 


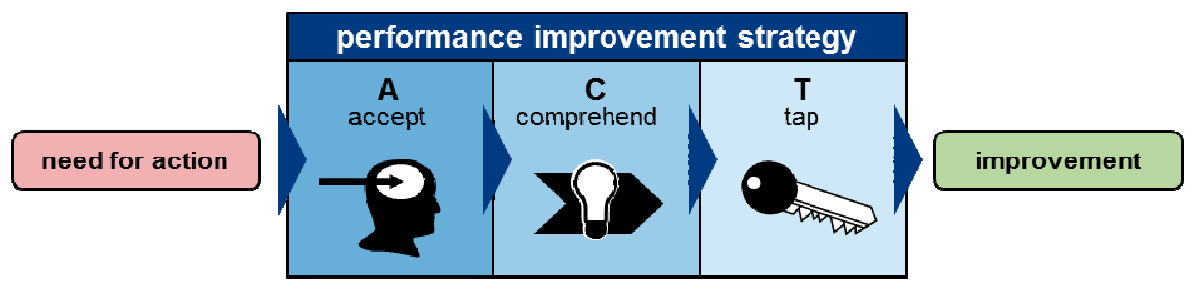

Fig. 3. ACT-concept to improve the performance of VPS in SMEs

The ACT-concept distinguishes three stages:

- First, accept the need for action: Process improvement always goes along with change. To accept this change, the company must be aware of the need for action. In this stage, intuitive diagrams visualize the need for action without being too detailed.

- Second, comprehend the need for action: After accepting the need for action, the question will be what to change in detail. This is answered by showing the company's strengths and weaknesses for each action element.

- Third, tap the need for action: Adequate measures are needed to overcome the weaknesses. Therefore company-specific measures are recommended. These measures are detailed in measure-profiles, which deliver the necessary background for the SME.

The ACT-concept motivates the SME to deal more intensive with VPS and to identify and exploit all benefits of virtual engineering. In the following we detail the three stages of ACT.
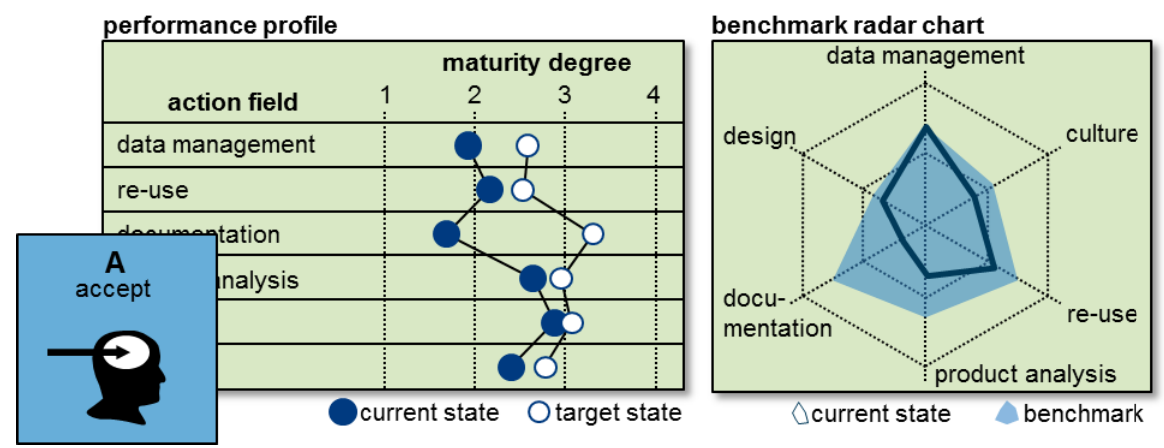

Fig. 4. Stage ACCEPT of the ACT-concept

Stage ACCEPT visualizes the need for action in diagrams (figure 4). The performance profile displays the user's maturity level for each action field. This allows a quick overview of the current performance. In addition, the target state is displayed. The target state is determined individually from the company-class. From the distance between current state and target state the need for action can be derived. The bench- 
mark radar chart shows the performance of the company in comparison to the performance of other companies. These striking visualizations should be used to convince the management of expanding the use of VPS.

Stage COMPREHEND details the need for action (figure 5). Therefore, the action elements in which the SME does not reach the recommended performance levels are displayed. Icons indicate whether the current state differs from the target state. Information from this stage is particularly relevant for persons, who are concerned with performance improvement in product development, e.g. the development manager. This group of people needs a thorough understanding of the strengths and weaknesses of the organization.

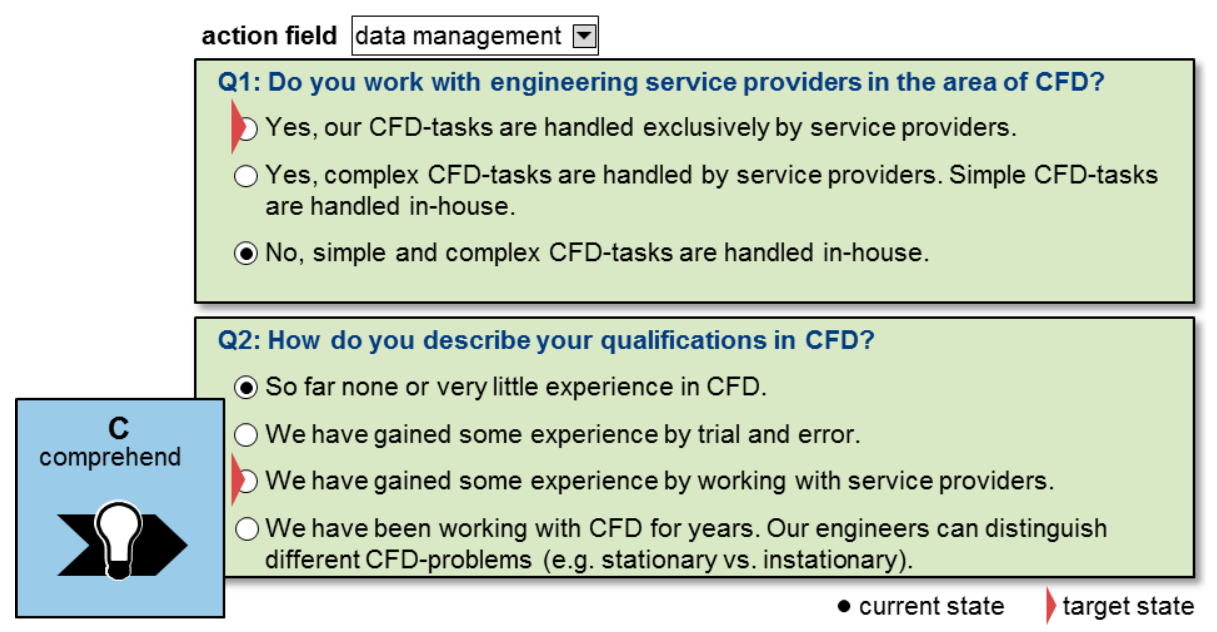

Fig. 5. Stage COMPREHEND of the ACT-concept

As SMEs should only aspire economically advantageous performance levels, it is important that the need for action is derived from the company's individual target state. We emphasize this with an example. Two times per year an SME is challenged by the integration of a dryer into his system. As the drying process is of crucial importance for product quality, the company decides to buy a suitable $\mathrm{CFD}^{3}$-tool and to simulate the process in-house. It quickly recognizes that the effort for practice is very high. In addition, the simulation results deviate from reality, because the boundary parameters are not defined properly. Simulating the second dryer a few months later, it can barely remember the operation of the CFD-tool. It decides to quit working with the tool. The investment in software and many staff hours are lost. Besides the economic loss also the trust in VPS suffers, since the SME now decides that CFD is too complex and unnecessary. In this case, the SME would have been more successful, if it would have started on a lower performance level. It should have engaged an external service provider for simulation. So it can gain experience in the field of CFD without taking risks.

${ }^{3}$ Computational Fluid Dynamics (CFD) means the computer-aided simulation of fluid mechanics [21]. 
The step TAP shows a possible way to close the gap to the target state (figure 6). The comparison between maturity level and target state allows deriving concrete measures for performance improvement. These measures are presented in measureprofiles. As most SMEs require a lot of measures, a simultaneous implementation of all measures often is neither economical nor organizational reasonable. Therefore, the cost-benefit-ratio allows a prioritization of measures.

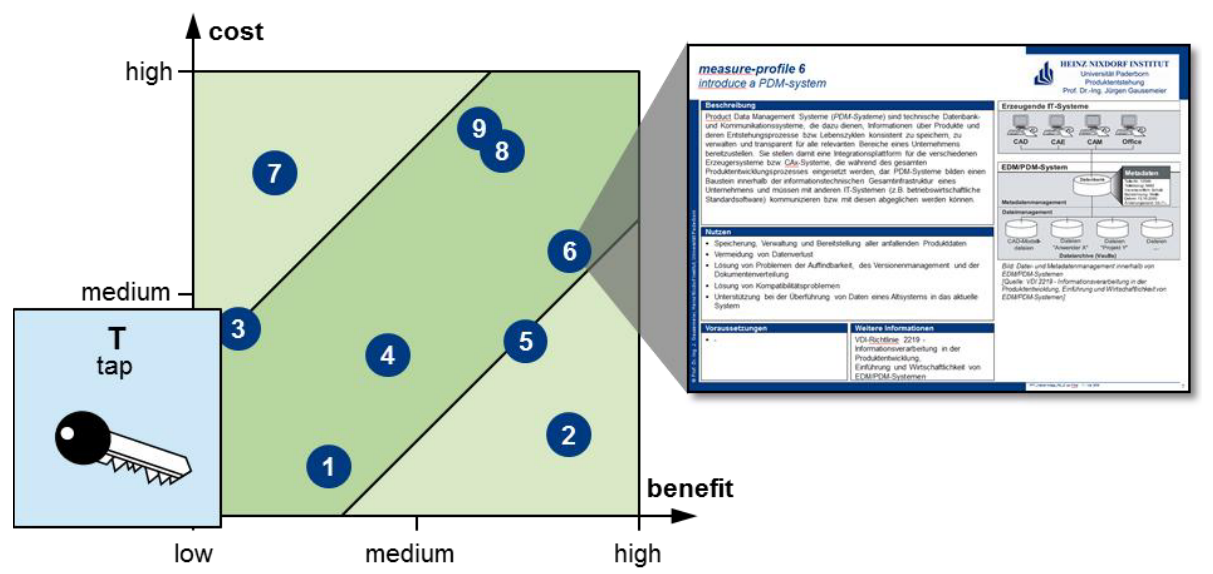

Fig. 6. Stage TAP of the ACT-concept

\section{$5 \quad$ Conclusion and Outlook}

The paper started with a brief overview on existing approaches for performance evaluation and improvement. We pointed out that specific requirements arise out of the need for maturity models in SMEs. SMEs neither have the time nor the money to get into complex models for performance improvement, such as CMMI (Capability Maturity Model Integration). We introduced an approach for maturity based process improvement that is suitable for SMEs and focused on virtual engineering.

The approach achieves all the specific requirements that arise out of the need for maturity models in SMEs. The interactive questionnaire supports the performance evaluation and is easily applicable. Due to the fact that the questionnaire is internet based no special software is required. Additionally, only a short time for application is necessary. The questionnaire can be completed in not more than two days. Through the prepared expert knowledge it is based on best practices and no external consultants are necessary. The analysis of influences defines an economical company-specific target state. Concrete measures for performance improvement are recommended.

The developed maturity model offers a step by step performance improvement to SMEs. The ACT-concept motivates the SME to deal more intensive with VPS and to exploit its benefits. In Future works, the maturity model will be validated in SMEs. Therefore we will compare the results of the VPS-Benchmark with the results of consultants. 
Acknowledgement. This contribution was developed in the course of the project "Virtual Prototyping and Simulation Benchmark: tools for analysing and optimizing the use of virtual prototyping and simulation in the product development process" funded by the Ziel-2 programme "Hightech.NRW" of North Rhine-Westphalia. This is a part of ERDF: European Regional Development Fund.

\section{References}

1. Rix, J.: Virtual Prototyping - Virtual Environments and the Product Design Process. Chapman \& Hall, London (1995)

2. Gausemeier, J., Berssenbrügge, J., Grafe, M., Kahl, S., Wassmann, H.: Design and VR/AR-based Testing of Advanced Mechatronic Systems. In: Proceedings of the 2nd Sino-German-Workshop, Virtual and Augmented Reality in Industry. Springer, Shanghai (2009)

3. Stark, J.: Product Lifecycle Management - 21st Century Paradigm for Product Realisation. Springer, London (2011)

4. Fraser, P., Moultrie, J., Gregory, M.: The use of maturity models/grids as a tool in assessing product development capability. In: Proceedings of the IEEE Engineering Management Conference IEMC, Cambridge (2002)

5. Spur, G., Krause, F.-L.: Das virtuelle Produkt. Management der CAD-Technik. Carl Hanser Verlag, München (1997)

6. Knoblinger, C., Oehmen, J., Rebentisch, E., Seering, W., Helten, K.: Requirements for product development self-assessment tools. In: Proceedings of the 18th International Conference on Engineering Design (ICED 2011), Copenhagen (2011)

7. de Bruin, T., Rosemann, M., Freeze, R., Kulkarni, U.: Understanding the Main Phases of Developing a Maturity Assessment Model. In: Proceedings of the 6th Australasian Conference on Information Systems, ACIS (2005)

8. Christiansen, S.-K.: Methode zur Klassifikation und Entwicklung reifegradbasierter Leistungsbewertungs- und Leistungssteigerungsmodelle. HNI-Verlagsschriftenreihe, Paderborn (2009)

9. Kohlegger, M., Maier, R., Thalmann, S.: Understanding Maturity Models - Results of a Structured Content Analysis. In: Proceedings of I-KNOW and I-SEMANTICS, Graz (2009)

10. Maier, A., Moultrie, J., Clarkson, J.: Developing maturity grids for assessing organisational capabilities: Practitioner guidance. In: Proceedings of the 4th International Conference on Management Consulting, Academy of Management, Vienna (2009)

11. Harmon, P.: Process Maturity Models. BPTrends, Email Advisor, vol. 5 (March 2009), http: / /www. bptrends. com

12. Batenburg, R., Helms, R., Versendaal, J.: PLM roadmap: stepwise PLM implementation based on the concepts of maturity and alignment. In: Proceedings of the International Journal of Product Lifecycle Management (2006)

13. Software Engineering Institute (SEI), CMMI® for Development, Version 1.2 (August 2006), http: / / www. sei.cmu. edu

14. Chrissis, M.B., Konrad, M., Shrum, S.: CMMI. Guidelines for Process Integration and Product Improvement. Addison-Wesley Longman, Amsterdam (2003)

15. Hammer, M.: The Process Audit. Harvard Business Review (April 2007)

16. Mettler, T.: A Design Science Research Perspective on Maturity Models in Information Systems, Technical Report BE IWI/HNE/03, Universität St. Gallen, St. Gallen (2009) 
17. Van Looy, A., De Backer, M., Poels, G.: Which Maturity Is Being Measured? A Classification of Business Process Maturity Models. In: Proceedings of the 5th SIKS/BENAIS Conference on Enterprise Information Systems, Eindhoven (2010)

18. Röglinger, M., Pöppelbuß, J.: What makes a useful maturity model? A framework for general design principles for maturity models and its demonstration in business process management. In: Proceedings of the 19th European Conference on Information Systems (ECIS 2011), Association for Information Systems (AIS), Paper 28 (June 2011)

19. Röglinger, M., Pöppelbuß, J., Becker, J.: Maturity Models in Business Process Management. Business Process Management Journal, Discussion Paper WI-352 (2011)

20. Gausemeier, J., Bensiek, T., Kuehn, A., Grafe, M.: Maturity based improvement of product development processes in small and medium-sized enterprises. In: 12th International Design Conference - DESIGN 2012, Dubrovnik, Croatia, May 21-24 (2012)

21. Wendt, J.F., Anderson, J., Dick, E., Degrez, G., Degroote, J., Grundmann, R., Vierendeels, J.: Compuational Fluid Dynamics - an introduction. Springer, Berlin (2009) 